MATHEMATICS OF COMPUTATION

Volume 76, Number 260, October 2007, Pages 2087-2094

S 0025-5718(07)01955-2

Article electronically published on April 17, 2007

\title{
A SEARCH FOR FIBONACCI-WIEFERICH AND WOLSTENHOLME PRIMES
}

\author{
RICHARD J. MCINTOSH AND ERIC L. ROETTGER
}

\begin{abstract}
A prime $p$ is called a Fibonacci-Wieferich prime if $F_{p-\left(\frac{p}{5}\right)} \equiv 0$ $\left(\bmod p^{2}\right)$, where $F_{n}$ is the $n$th Fibonacci number. We report that there exist no such primes $p<2 \times 10^{14}$. A prime $p$ is called a Wolstenholme prime if $\left(\begin{array}{c}2 p-1 \\ p-1\end{array}\right) \equiv 1\left(\bmod p^{4}\right)$. To date the only known Wolstenholme primes are 16843 and 2124679 . We report that there exist no new Wolstenholme primes $p<10^{9}$. Wolstenholme, in 1862 , proved that $\left(\begin{array}{c}2 p-1 \\ p-1\end{array}\right) \equiv 1\left(\bmod p^{3}\right)$ for all primes $p \geq 5$. It is estimated by a heuristic argument that the "probability" that $p$ is Fibonacci-Wieferich (independently: that $p$ is Wolstenholme) is about $1 / p$. We provide some statistical data relevant to occurrences of small values of the Fibonacci-Wieferich quotient $F_{p-\left(\frac{p}{5}\right)} / p$ modulo $p$.
\end{abstract}

\section{INTRODUCTION}

Let $P$ and $Q$ be nonzero integers. The Lucas sequences $\left\{U_{n}\right\}=\left\{U_{n}(P, Q)\right\}$ and $\left\{V_{n}\right\}=\left\{V_{n}(P, Q)\right\}$ associated to the pair $(P, Q)$ are defined by

$$
U_{0}=0, U_{1}=1, U_{n+1}=P U_{n}-Q U_{n-1} \text { for } n \geq 1,
$$

and

$$
V_{0}=2, V_{1}=P, V_{n+1}=P V_{n}-Q V_{n-1} \text { for } n \geq 1 .
$$

The discriminant of the characteristic polynomial of these sequences is given by $D=P^{2}-4 Q$. We will assume that $D \neq 0$. The special case $P=-Q=1$ defines the well-known Fibonacci sequence, which we will denote by $\left\{F_{n}\right\}=\left\{U_{n}(1,-1)\right\}$, and the Lucas numbers usually denoted by $\left\{L_{n}\right\}=\left\{V_{n}(1,-1)\right\}$.

It is well-known (see [5, pp. 393-395]) that $F_{p-\left(\frac{p}{5}\right)}$ is divisible by $p$, where $p$ is prime and $\left(\frac{p}{5}\right)$ denotes the Legendre symbol. If $F_{p-\left(\frac{p}{5}\right)}$ is divisible by $p^{2}$, then we call $p$ a Fibonacci-Wieferich prime (these primes are sometimes called Wall-SunSun primes [3, pp. 110-112]). A Wieferich prime is a prime $p$ satisfying $2^{p-1} \equiv 1$ $\left(\bmod p^{2}\right)$.

According to the most recent search 9 ] the only Wieferich primes $p<1.25 \times 10^{15}$ are 1093 and 3511. Williams [19] found no Fibonacci-Wieferich primes below $10^{9}$ and Montgomery [11 extended this to $2^{32}$. Our computer search found no such primes below $2 \times 10^{14}$. Of historical interest is the connection between FibonacciWieferich primes and Fermat's Last Theorem. Sun and Sun [17] proved that if

Received by the editor June 14, 2005 and, in revised form, May 19, 2006.

2000 Mathematics Subject Classification. Primary 11A07, 11A41, 11B39, 11 Y99.

Key words and phrases. Fibonacci number, Wieferich prime, Wall-Sun-Sun prime, Wolstenholme prime.

(C)2007 American Mathematical Society Reverts to public domain 28 years from publication 
the first case of Fermat's Last Theorem fails for the exponent $p$, then $p$ must be a Fibonacci-Wieferich prime.

\section{LUCAS-WIEFERICH PRIMES}

Returning to the general Lucas Sequences $\left\{U_{n}(P, Q)\right\}$ and $\left\{V_{n}(P, Q)\right\}$, we have $U_{p-\left(\frac{D}{p}\right)}$ divisible by $p$ whenever $p$ is a prime not dividing $2 Q$. Let $\epsilon$ denote the Legendre symbol $\left(\frac{D}{p}\right)$, where $D=P^{2}-4 Q$. The modulo $p$ residues of $U_{p-\epsilon}, V_{p-\epsilon}$, $U_{p}$ and $V_{p}$ are given by the following congruences, valid for primes $p$ not dividing $2 Q D$ :

$$
\begin{aligned}
& U_{p-\epsilon} \equiv 0 \quad(\bmod p), \\
& V_{p-\epsilon} \equiv 2 Q^{(1-\epsilon) / 2} \quad(\bmod p), \\
& U_{p} \equiv \epsilon \quad(\bmod p), \\
& V_{p} \equiv P \quad(\bmod p) .
\end{aligned}
$$

For a detailed discussion of these and many other congruences for Lucas sequences we refer the reader to Ribenboim [14] and Riesel [15].

Congruence $(2)$ enjoys the following $\left(\bmod p^{2}\right)$ extension.

Lemma. If the prime $p$ does not divide $2 Q D$, then

$$
V_{p-\epsilon} \equiv Q^{(1-\epsilon) / 2}\left(Q^{p-1}+1\right) \quad\left(\bmod p^{2}\right) .
$$

Proof. We begin with equations (IV.4) with $n=m$ and (IV.5) in [14, p. 57]:

$$
2 V_{2 m}=V_{m}^{2}+D U_{m}^{2}
$$

and

$$
V_{2 m}=V_{m}^{2}-2 Q^{m}
$$

Subtracting (7) from (6) yields

$$
V_{2 m}=D U_{m}^{2}+2 Q^{m} .
$$

Let $2 m=p-\epsilon$ and $\mu=\left(\frac{Q}{p}\right)$ denote the Legendre symbol. By [14, p. 63], statement (IV.23), we have $p \mid U_{m}$ if $\mu=1$ and $p \mid V_{m}$ if $\mu=-1$. Therefore, it follows from (8) if $\mu=1$ and from (7) if $\mu=-1$ that

$$
V_{p-\epsilon}=V_{2 m} \equiv 2 \mu Q^{m}=2 \mu Q^{(p-\epsilon) / 2}=2 \mu Q^{(p-1) / 2} Q^{(1-\epsilon) / 2} \quad\left(\bmod p^{2}\right) .
$$

Since $Q^{(p-1) / 2} \equiv \mu(\bmod p)$ by Euler's criterion, we have $Q^{(p-1) / 2} \equiv \mu(1+a p)$ $\left(\bmod p^{2}\right)$ for some integer $a$. Hence $Q^{p-1} \equiv 1+2 a p\left(\bmod p^{2}\right)$, and therefore $2 \mu Q^{(p-1) / 2} \equiv 2+2 a p \equiv Q^{p-1}+1\left(\bmod p^{2}\right)$. Finally, by (9) we obtain $V_{p-\epsilon} \equiv$ $\left(Q^{p-1}+1\right) Q^{(1-\epsilon) / 2}\left(\bmod p^{2}\right)$, which completes the proof of $(5)$.

Sometimes $U_{p-\epsilon}$ is divisible by $p^{2}$. We call these primes Lucas-Wieferich primes associated to the pair $(P, Q)$. Every Wieferich prime is a Lucas-Wieferich prime associated to the pair $(3,2)$. Equivalent congruences for Lucas-Wieferich primes are given in Theorem 1 .

Theorem 1. If the prime $p$ does not divide $2 P Q D$, then the following are equivalent:

(i) $U_{p-\epsilon} \equiv 0\left(\bmod p^{2}\right)$,

(ii) $V_{p-\epsilon} \equiv 2 \mu Q^{(p-\epsilon) / 2}\left(\bmod p^{4}\right)$, 
(iii) $V_{p-\epsilon} \equiv 2 \mu Q^{(p-\epsilon) / 2}\left(\bmod p^{3}\right)$,

(iv) $U_{p} \equiv \epsilon\left(Q^{p-1}+1\right) / 2\left(\bmod p^{2}\right)$,

(v) $V_{p} \equiv P\left(Q^{p-1}+1\right) / 2\left(\bmod p^{2}\right)$.

Proof. We will first show that (i) $\Rightarrow$ (ii) $\Rightarrow$ (iii) $\Rightarrow$ (i).

(i) $\Rightarrow$ (ii). Suppose that $U_{p-\epsilon} \equiv 0\left(\bmod p^{2}\right)$. By [14, p. 56], equation (IV.1), we have $V_{p-\epsilon}^{2}-4 Q^{p-\epsilon}=D U_{p-\epsilon}^{2}$. Hence $\left(V_{p-\epsilon}-2 \mu Q^{(p-\epsilon) / 2}\right)\left(V_{p-\epsilon}+2 \mu Q^{(p-\epsilon) / 2}\right)=$ $V_{p-\epsilon}^{2}-4 Q^{p-\epsilon}=D U_{p-\epsilon}^{2} \equiv 0\left(\bmod p^{4}\right)$, where $\mu=\left(\frac{Q}{p}\right)$ denotes the Legendre symbol. By (9) we have $V_{p-\epsilon}-2 \mu Q^{(p-\epsilon) / 2} \equiv 0(\bmod p)$, which implies that $V_{p-\epsilon}+2 \mu Q^{(p-\epsilon) / 2} \not \equiv 0(\bmod p)$ since $p$ does not divide $2 Q$. Therefore $V_{p-\epsilon}-$ $2 \mu Q^{(p-\epsilon) / 2} \equiv 0\left(\bmod p^{4}\right)$, and so $V_{p-\epsilon} \equiv 2 \mu Q^{(p-\epsilon) / 2}\left(\bmod p^{4}\right)$.

(ii) $\Rightarrow$ (iii) is trivial.

(iii) $\Rightarrow(\mathrm{i})$. Suppose that $V_{p-\epsilon} \equiv 2 \mu Q^{(p-\epsilon) / 2}\left(\bmod p^{3}\right)$. Then $V_{p-\epsilon}^{2}-4 Q^{p-\epsilon}=$ $\left(V_{p-\epsilon}-2 \mu Q^{(p-\epsilon) / 2}\right)\left(V_{p-\epsilon}+2 \mu Q^{(p-\epsilon) / 2}\right) \equiv 0\left(\bmod p^{3}\right)$ and so by [14, p. 56], equation (IV.1), it follows that $D U_{p-\epsilon}^{2}=V_{p-\epsilon}^{2}-4 Q^{p-\epsilon} \equiv 0\left(\bmod p^{3}\right)$. Since $p$ does not divide $D$ we obtain (i).

Next, we will prove that (i) and (iv) are equivalent. By [14, p. 56], equation (IV.2), we have $V_{n}=U_{n+1}-Q U_{n-1}$. Using the recurrence $U_{n+1}=P U_{n}-Q U_{n-1}$ we obtain

$$
V_{n}=2 U_{n+1}-P U_{n}
$$

and

$$
V_{n}=P U_{n}-2 Q U_{n-1} .
$$

Setting $n=p-1$ in (10) and $n=p+1$ in (11) we get

$$
V_{p-1}=2 U_{p}-P U_{p-1}
$$

and

$$
V_{p+1}=P U_{p+1}-2 Q U_{p} .
$$

Using (12) when $\epsilon=1$ and (13) when $\epsilon=-1$ we obtain

$$
V_{p-\epsilon}=-\epsilon P U_{p-\epsilon}+2 \epsilon Q^{(1-\epsilon) / 2} U_{p} .
$$

By (5) we have $V_{p-\epsilon} \equiv Q^{(1-\epsilon) / 2}\left(Q^{p-1}+1\right)\left(\bmod p^{2}\right)$. Therefore

$$
2 Q^{(1-\epsilon) / 2} U_{p}-P U_{p-\epsilon} \equiv \epsilon Q^{(1-\epsilon) / 2}\left(Q^{p-1}+1\right) \quad\left(\bmod p^{2}\right) .
$$

Since $p$ does not divide $2 P Q$ the equivalence of (i) and (iv) follows from the above congruence.

Finally, we will prove that (i) and (v) are equivalent. By [14, p. 56], equation (IV.2), we have $D U_{n}=V_{n+1}-Q V_{n-1}$. Using the recurrence $V_{n+1}=P V_{n}-Q V_{n-1}$ we obtain

$$
D U_{n}=2 V_{n+1}-P V_{n}
$$

and

$$
D U_{n}=P V_{n}-2 Q V_{n-1} .
$$

Setting $n=p-1$ in (14) and $n=p+1$ in (15) we get

$$
D U_{p-1}=2 V_{p}-P V_{p-1}
$$


and

$$
D U_{p+1}=P V_{p+1}-2 Q V_{p} .
$$

Using (16) when $\epsilon=1$ and (17) when $\epsilon=-1$ we obtain

$$
D U_{p-\epsilon}=-\epsilon P V_{p-\epsilon}+2 \epsilon Q^{(1-\epsilon) / 2} V_{p} .
$$

By (5) we have $V_{p-\epsilon} \equiv Q^{(1-\epsilon) / 2}\left(Q^{p-1}+1\right)\left(\bmod p^{2}\right)$. Therefore

$$
2 Q^{(1-\epsilon) / 2} V_{p}-\epsilon D U_{p-\epsilon} \equiv P Q^{(1-\epsilon) / 2}\left(Q^{p-1}+1\right) \quad\left(\bmod p^{2}\right)
$$

Since $p$ does not divide $2 Q D$ the equivalence of (i) and (v) follows from the above congruence. The proof of Theorem 1 is now complete.

Sun [16] showed that $p$ is a Fibonacci-Wieferich prime if and only if $L_{p-\epsilon} \equiv 2 \epsilon$ $\left(\bmod p^{4}\right)$, which proves that (i) and (ii) are equivalent when $P=-Q=1$.

\section{Algorithm for the FibonacCi-Wieferich prime SEARCh}

In general there is no known way to resolve $F_{p-\left(\frac{p}{5}\right)}\left(\bmod p^{2}\right)$, other than through explicit computations. From the recurrence $F_{n+1}=F_{n}+F_{n-1}$ we obtain

$$
\left(\begin{array}{c}
F_{n+1} \\
F_{n}
\end{array}\right)=\left(\begin{array}{ll}
1 & 1 \\
1 & 0
\end{array}\right)\left(\begin{array}{c}
F_{n} \\
F_{n-1}
\end{array}\right)=\left(\begin{array}{ll}
1 & 1 \\
1 & 0
\end{array}\right)^{n}\left(\begin{array}{l}
F_{1} \\
F_{0}
\end{array}\right)=\left(\begin{array}{ll}
1 & 1 \\
1 & 0
\end{array}\right)^{n}\left(\begin{array}{l}
1 \\
0
\end{array}\right)
$$

Powers of the above matrix are computed $\left(\bmod p^{2}\right)$ by a standard binary power ladder. Since our 64-bit processors cannot handle products of magnitude $p^{4}$, we invoked base $p$ representations and thereby "split" the multiplication of two numbers $\left(\bmod p^{2}\right)$. Every $x=a+b p\left(\bmod p^{2}\right)$ is represented by $\{a, b\}$, with both $a, b$ always reduced $(\bmod p)$. To avoid repeated use of long division by $p$ we computed and stored the value of $1 / p$ in double precision floating-point. The quotient $k / p$ is given by $\lfloor 0.5+k(1 / p)\rfloor$ and the remainder is given by $k-\lfloor 0.5+k(1 / p)\rfloor$. If the remainder is negative, then $p$ is added to the remainder and 1 is subtracted from the quotient. Crandall [3, pp. 9-10] calls this "steady-state division". Overflow errors do not occur when $p<4 \times 10^{14}$ and $k<p^{2}$. The final value of $F_{p-\left(\frac{p}{5}\right)} \equiv$ $B+A p\left(\bmod p^{2}\right)$ is represented by $\{B, A\}$, where $0 \leq B<p$ and $-p / 2<A<p / 2$. If $B \neq 0$, our program exits with fatal error. Instances of $|A| \leq 100$ are recorded (see Table 1 below) for testing purposes and statistical analysis. The value $A=0$ would signify a Fibonacci-Wieferich prime. Extremely close calls $(A= \pm 1)$ occur when $p=2,3,5,17,251,733,1063,123863$ and 1677209 .

We were interested in applying the above test only to actual primes $p$. Due to the nature of binary power ladder we found it wasteful to employ the Fermat test, that is, whether $2^{p-1} \equiv 1(\bmod p)$, for selecting primes. Instead we used an incremental sieve designed by Crandall [3, p. 100] which sieves the integers in blocks of a million at a time.

Most of the computation was performed on an SGI Onyx/2 server with 24 R12000 processors and $12 \mathrm{~GB}$ of RAM, running IRIX 6.5. Near the end of the search range each processor was capable of processing an interval of $45 \times 10^{9}$ integers per day or about 16000 primes per second. About $10 \%$ of the total running time was used for sieving the primes. Taking advantage of 64 -bit processors our program was about 7 times faster than the program used in $[9$ which was designed for 32-bit processors. 
TABLE 1. Instances of $F_{p-\left(\frac{p}{5}\right)} \equiv A p\left(\bmod p^{2}\right)$ with $|A| \leq 100$ and $2^{32}<p<2 \times 10^{14}$

\begin{tabular}{|r|r|}
\hline \multicolumn{1}{|c|}{$p$} & \multicolumn{1}{|c|}{$A$} \\
\hline 6627557731 & -43 \\
6741860329 & 80 \\
6859114489 & 55 \\
8352762221 & 94 \\
9760159421 & -30 \\
10115341939 & 70 \\
10536116749 & 66 \\
10612008943 & -69 \\
11002117921 & 95 \\
11025166637 & 23 \\
12216759923 & -33 \\
12387724249 & 39 \\
13585864301 & 57 \\
13699540891 & 73 \\
13941800291 & 15 \\
16368086681 & 50 \\
16548311011 & 92 \\
17370126353 & -70 \\
18526398173 & -29 \\
20488487861 & -92 \\
24178397183 & 3 \\
25049632411 & 51 \\
31811337589 & -89 \\
37883499127 & -10 \\
50261755937 & -73 \\
65543096747 & -36 \\
74176637257 & -70 \\
82202291813 & -11 \\
87918267869 & 78 \\
\hline & \\
\hline
\end{tabular}

\begin{tabular}{|r|r|}
\hline$p$ & \multicolumn{1}{|c|}{$A$} \\
\hline 89845144679 & 51 \\
94903475011 & -15 \\
101876918491 & 87 \\
110717352637 & -77 \\
111646394549 & -86 \\
115301883659 & 60 \\
115364673283 & 62 \\
129316722167 & -22 \\
134431860461 & -30 \\
166466703223 & 64 \\
170273590301 & 78 \\
233642484991 & 89 \\
277764184829 & 64 \\
283750593739 & 37 \\
300258464153 & 70 \\
334015396151 & 79 \\
442650398821 & 74 \\
458432241569 & -61 \\
621291852133 & 96 \\
762383958397 & -86 \\
766193665711 & -8 \\
80053716979 & -20 \\
1082150673011 & -57 \\
1171853196853 & -30 \\
1551559563569 & 4 \\
1786416720937 & -82 \\
1996100161327 & 98 \\
2669682790919 & -10 \\
3311519272973 & -47 \\
\hline
\end{tabular}

\begin{tabular}{|r|r|}
\hline \multicolumn{1}{|c|}{$p$} & \multicolumn{1}{|c|}{$A$} \\
\hline 3814438808399 & -56 \\
3858738583171 & 44 \\
5457214172491 & 76 \\
8030311150847 & -12 \\
10591568377751 & 33 \\
11406840440243 & 26 \\
11456600879363 & -41 \\
12801531958729 & 17 \\
13860200708287 & -5 \\
18801391545961 & 33 \\
21960966892313 & 54 \\
22548139284371 & -16 \\
25186595067349 & -59 \\
26861987497291 & -28 \\
28263796914043 & -88 \\
39568597208207 & 53 \\
46006966741789 & -59 \\
64241561031937 & -44 \\
67721845179979 & -52 \\
75320741942123 & 71 \\
82789107950701 & -42 \\
85136199318719 & -92 \\
86040142362653 & 19 \\
88536418898561 & 99 \\
90299689540433 & 56 \\
91486955300761 & -71 \\
106692167197171 & -85 \\
155979357644989 & 27 \\
\hline
\end{tabular}

\section{Statistical Considerations}

Are there any Fibonacci-Wieferich primes? Since $A=A(p)$ is an integer in the interval $(-p / 2, p / 2)$, one might assume that the "probability" of $A$ taking on any particular value, say the value 0 , is equal to $1 / p$. One might view the FibonacciWieferich test for different primes as "independent" events. Therefore, by this heuristic argument the number of Fibonacci-Wieferich primes in an interval $[x, y]$ is expected to be

$$
\sum_{x \leq p \leq y} \frac{1}{p} \approx \sum_{n=x}^{y} \frac{1}{n \ln n} \approx \int_{x}^{y} \frac{d t}{t \ln t}=\ln (\ln y)-\ln (\ln x),
$$

since it follows from the Prime Number Theorem that the "probability" that an integer $p$ is prime is about $1 / \ln p$. If this is the case, then the expected number of Fibonacci-Wieferich primes in the interval $\left[2^{32}, 2 \times 10^{14}\right]$ is approximately 0.395 , 
and so there was indeed a fairly good chance to find a Fibonacci-Wieferich prime, which justifies the effort in undertaking this search.

If we take into account "near" Fibonacci-Wieferich primes, that is, primes $p$ with $|A| \leq 100$, then the expected number of "close calls" in the above interval is approximately $201 \times 0.395 \approx 79.4$. From Table 1 we see that the actual count is 86 .

\section{WOLSTENHOLME PRIMES}

In 1862 Wolstenholme [20] (also see [5, p. 271] and [14, p. 29]) proved that $\left(\begin{array}{c}2 n-1 \\ n-1\end{array}\right) \equiv 1\left(\bmod n^{3}\right)$ for all primes $n \geq 5$. McIntosh [10] found no composite solutions $n<10^{9}$, and it is conjectured that there are none [7, problem B31]. Unlike that of Wilson's Theorem the converse of Wolstenholme's Theorem is a very difficult problem.

The term Wolstenholme prime has been introduced in [10, for primes $p$ satisfying the congruence $\left(\begin{array}{c}2 p-1 \\ p-1\end{array}\right) \equiv 1\left(\bmod p^{4}\right)$. In $[10, p .386]$ the following theorem was proved, where $B_{n}$ denotes the $n$th Bernoulli number.

Theorem 2. For all primes $p \geq 11$ the following are equivalent:

(i) $p$ is a Wolstenholme prime,

(ii) $p$ divides the numerator of $B_{p-3}$,

(iii) $\sum_{p / 6<k<p / 4} \frac{1}{k^{3}} \equiv 0(\bmod p)$.

Statement (ii) appears in a criterion concerning Fermat's Last Theorem. A prime $p$ is regular if and only if $p$ does not divide the numerators of the Bernoulli numbers $B_{2}, B_{4}, \ldots, B_{p-3}$. For all such primes the algebraic proof of Fermat's Last Theorem is valid (see [6, p. 244] and [13, p. 10]). This was one of the main reasons for the search of irregular primes. Buhler, Crandall, Ernvall, and Metsänkylä [1] calculated all irregular primes up to $4 \times 10^{6}$ by evaluating sums of like powers of numbers in arithmetic progression, and these calculations were extended in 2 by use of different methods to $12 \times 10^{6}$.

The Wolstenholme primes are those irregular primes where $p$ divides the numerator of $B_{p-3}$. The only known Wolstenholme primes are 16843 and 2124679. The first was found (though not explicitly reported) by Selfridge and Pollak (Notices AMS 11 (1964), 97), and later confirmed by W. Johnson [8] and S.S. Wagstaff (Notices AMS 23 (1976), A-53). The second was found by J. Buhler, R. Crandall, R. Ernvall, and T. Metsänkylä [1, and later, independently, by McIntosh [10, p. 387 ] in his search up to $2 \times 10^{8}$. Using (iii) we extended the search to $10^{9}$ and found no new Wolstenholme primes, and thus no new primes $p$ divide the numerator of $B_{p-3}$.

Our program, written by Montgomery [12, evaluates the least two significant coefficients $c_{0}$ and $c_{1}(\bmod p)$ in the polynomial

$$
f(x)=\prod_{p / 6<k<p / 4}\left(x+k^{3}\right)
$$

by the use of a polynomial scheme similar to the one developed in [4, p. 441]. The sum $(\bmod p)$ of the reciprocals of the roots of $f(x)$ is given by $-c_{1} / c_{0}$. The value $c_{1}=0$ would signify a Wolstenholme prime. The fast Fourier transform (FFT) and the Nussbaumer convolution were not used. Most of the computation was performed on an SGI Onyx/2 server with 24 R12000 processors and 12 GB 
of RAM, running IRIX 6.5. Near the end of the search range each processor was capable of processing an interval of 412000 integers per day or about 4.3 seconds per prime. The Fermat test, that is, whether $2^{p-1} \equiv 1(\bmod p)$, was used for selecting primes. Due to the relatively long time required to process each prime the use of a sieve for selecting primes would not offer a significant reduction in total running time.

\section{ACKNOWLEDGMENTS}

The authors are grateful to Richard Crandall, Joshua Knauer and Peter Montgomery for their help in the search for Fibonacci-Wieferich and Wolstenholme primes. Support by the Natural Sciences and Engineering Research Council of Canada is gratefully acknowledged.

\section{REFERENCES}

[1] J. Buhler, R. Crandall, R. Ernvall, and T. Metsänkylä, Irregular primes and cyclotomic invariants to four million, Math. Comp 61 (1993) 151-153. MR1197511 (93k:11014)

[2] J. Buhler, R. Crandall, R. Ernvall, T. Metsänkylä, and M.A. Shokrollahi, Irregular primes and cyclotomic invariants to 12 million, J. Symbolic Comput 31 (2001) 89-96. MR1806208 (2001m:11220)

[3] R.E. Crandall, Topics in Advanced Scientific Computation, TELOS/Springer-Verlag, Santa Clara, CA, 1996. MR.1392472 (97g:65005)

[4] R.E. Crandall, K. Dilcher, and C. Pomerance, A search for Wieferich and Wilson primes, Math. Comp 66 (1997) 433-449. MR1372002 (97c:11004)

[5] L.E. Dickson, The History of the Theory of Numbers, vol. 1, Reprinted: Chelsea Publishing Company, New York, 1966.

[6] H.M. Edwards, Fermat's Last Theorem, Springer-Verlag, New York, 1977. MR616635 (83b:12001a)

[7] R.K. Guy, Unsolved Problems in Number Theory, Third ed., Springer-Verlag, New York, 2004. MR.2076335 (2005h:11003)

[8] W. Johnson, Irregular primes and cyclotomic invariants, Math. Comp 29 (1975) 113-120. MR0376606 (51:12781)

[9] J. Knauer and J. Richstein, The continuing search for Wieferich primes, Math. Comp $\mathbf{7 4}$ (2005) 1559-1563. MR2137018(2006a:11006)

[10] R.J. McIntosh, On the converse of Wolstenholme's Theorem, Acta Arith 71 (1995) 381-389. MR.1339137 (96h:11002)

[11] P. Montgomery, New solutions of $a^{p-1} \equiv 1\left(\bmod p^{2}\right)$, Math. Comp 61 (1991) 361-363. MR:1182246 (94d:11003)

[12] , private communication, 1993.

[13] P. Ribenboim, 13 Lectures on Fermat's Last Theorem, Springer-Verlag, New York, 1979. MR551363 (81f:10023)

[14] , The New Book of Prime Number Records, Third ed., Springer-Verlag, New York, 1996. MR1377060 (96k:11112)

[15] H. Riesel, Prime Numbers and Computer Methods for Factorization, Birkhäuser Publishers, Boston, 1985. MR897531 (88k:11002)

[16] Z.-H. Sun, private communication, 2005.

[17] Z.-H. Sun and Z.-W. Sun, Fibonacci numbers and Fermat's last theorem, Acta Arith 60 (1992) 371-388. MR.1159353 (93e:11025)

[18] D.D. Wall, Fibonacci series modulo m, Amer. Math. Monthly 67 (1960) 525-532. MR0120188 $(22: 10945)$

[19] H.C. Williams, The influence of computers in the development of number theory, Comput. Math. Appl 8 (1982) 75-93. MR649653 (83c:10002)

[20] J. Wolstenholme, On certain properties of prime numbers, Quart. J. Math 5 (1862) 35-39. 
Department of Mathematics and Statistics, University of Regina, Regina, SaskatcheWAN, CANADA S4S 0A2

E-mail address: mcintosh@math.uregina.ca

Department of Mathematics and Statistics, University of Calgary, Calgary, AlBERTA, CANADA T2N 1N4

E-mail address: roettgee@math.ucalgary.ca 